\title{
1 Role of theobromine in cocoa's metabolic properties in healthy rats
}

2 Mariona Camps-Bossacoma ${ }^{\dagger}$, Mar Garcia-Aloy , Sandra Saldaña-Ruiz ${ }^{\dagger}$, Trinitat Cambras ${ }^{\dagger}$, Raúl

3 González-Domínguez ${ }^{\ddagger}$, Àngels Franch ${ }^{\dagger}$, Francisco J. Pérez-Cano ${ }^{\dagger *}$, Cristina Andres-Lacueva ${ }^{\ddagger}$,

$4 \quad$ Margarida Castell ${ }^{\dagger}$

$5 \quad$ †Secció de Fisiologia, Departament de Bioquímica i Fisiologia, Facultat de Farmàcia i Ciències de

6 l'Alimentació, Universitat de Barcelona (UB), Barcelona; Institut de Recerca en Nutrició i

7 Seguretat Alimentària (INSA-UB), Barcelona, Spain

8 \$Biomarkers and Nutrimetabolomics Laboratory, Departament de Nutrició, Ciències de

9 l'Alimentació i Gastronomia, XaRTA, INSA-UB, Facultat de Farmàcia i Ciències de l'Alimentació,

10 UB, Barcelona; CIBER de Fragilidad y Envejecimiento (CIBERFES), Instituto de Salud Carlos III,

11 Barcelona, Spain

E-mails: marionacamps@ub.edu; margarcia@ub.edu; ssaldana1@hotmail.com; cambras@ub.edu;

13 raul.gonzalez@ub.edu; angelsfranch@ub.edu; franciscoperez@ub.edu; candres@ub-edu;

14 margaridacastell@ub.edu

15 *Address correspondence: franciscoperez@ub.edu; Tel.: +34 934024505 
Cocoa is rich in polyphenols and methylxanthines and it has been reported that its consumption, among other properties, has beneficial effects on metabolism. This study aimed to investigate the role of theobromine in cocoa's metabolic properties in healthy rats. In addition to morphometric measurements, biochemical markers of lipids and glucose metabolism and gene expression of molecules related to immune cells in adipose and hepatic tissues were assessed after 7 or 18 days of diet. Additionally, a metabolomic analysis was carried out at day 7 . This study revealed the presence of six discriminant metabolites in plasma due to the diets. Moreover, the results showed that theobromine is the main responsible factor for cocoa's effects on body weight gain as well as on lipid and glucose metabolism. The effects on body weight and lipids appeared as early as after 7 days of diet, whereas those affecting glucose metabolism required a longer intervention. 
Cocoa is a product obtained from the seeds of the Theobroma cacao L. tree, which contains fiber, proteins, carbohydrates, lipids, minerals, vitamins, polyphenols and methylxanthines ${ }^{1,2}$. Within polyphenols, cocoa mainly contains flavonoids, the most important ones being the flavan-3-ols, such as epicatechin, catechin and procyanidins. Otherwise, theobromine (3,7-dimethylxanthine) is the methylxanthine found in the highest concentration in $\operatorname{cocoa}^{3}$.

In recent years, cocoa has increasingly attracted attention because of its potential health effects ${ }^{4}$. Cocoa's anti-inflammatory, antioxidant, anti-allergy and anti-obesity properties have been reported; its intake also influences mood, intestinal microbiota, the cardiovascular system and, interestingly, the metabolic profile ${ }^{4-9}$. Within the role of cocoa in metabolic properties, it has been shown that cocoa consumption has a beneficial effect on obesity as well as in the improvement of glucose, lipids and insulin levels $8,10,11$. Preclinical studies evidenced that the consumption of a $10 \%$ cocoaenriched diet for 9 weeks in diabetic Zucker rats lowered glucose and insulin levels, and improved glucose tolerance and insulin resistance ${ }^{12}$. Similarly, the administration of $8 \%$ cocoa powder to mice with high-fat-diet-induced obesity reduced body weight $(\mathrm{BW})$ gain, and insulin resistance ${ }^{11}$. Likewise, mice fed with high-fat-diet treated with a cocoa polyphenol concentrate decreased adipose tissue mass and plasma triglycerides (TG), and consequently the BW gain, without modifications in plasma glucose and cholesterol levels ${ }^{13}$. Otherwise, obese diabetic rats fed a cocoa extract enriched with polyphenols and methylxanthines for 4 weeks showed reduced plasma total cholesterol, TG and low-density lipoprotein cholesterol ${ }^{14}$. Apart from the cocoa metabolic effects in rodents, interventional studies in humans have reinforced the role of cocoa in body metabolism. Thus, overweight/obese premenopausal women consuming a sugar-free natural cocoa beverage and dark chocolate twice a day for 18 weeks, decreased plasma glucose and insulin concentrations ${ }^{15}$, and a similar population consuming dark chocolate for 7 days had higher HDL cholesterol (HDL-c) and reduced the abdomen circumference ${ }^{16}$. Moreover, Martínez-López et al. reported that the 
consumption of a soluble cocoa product increased serum HDL-c whereas anthropometric parameters were unaffected ${ }^{17}$.

In our previous studies developed in rats, we detected that the administration of a diet containing $10 \%$ cocoa was associated with a lower BW gain ${ }^{18-21}$. More recently, we have shown that the attenuating effect on antibody production and the lowering of BW gain in rats fed cocoa were similar to that observed in rats receiving theobromine alone ${ }^{22}$. These results prompted us to consider whether theobromine may also contribute to the effects of cocoa intake on glucose and lipid metabolism. For this reason, the present study aimed to establish the role of theobromine in metabolic cocoa's properties. Apart from comparing the metabolic profile induced by cocoa and theobromine diets, rat physical activity was measured and an untargeted metabolomics study was carried out.

\section{MATERIALS AND METHODS}

\section{Reagents and biological material}

Cocoa powder was obtained from Idilia Foods S.L. (formerly Nutrexpa S.L., Barcelona, Spain), theobromine was purchased from Sigma-Aldrich (Madrid, Spain) and AIN-93M diet and basal mix by Harlan Teklad (Madison, USA). Ketamine was from Merial Laboratories S.A. (Barcelona, Spain) and xylazine from Bayer A.G. (Leverkusen, Germany). Polymerase Chain Reaction (PCR) TaqMan ${ }^{\circledR}$ primers were provided by Applied Biosystems (Weiterstadt, Germany). Rats were obtained from Janvier Labs (Saint-Berthevin Cedex, France).

\section{Experimental design}

Forty 3-wk-old Lewis rats were maintained at the animal facilities of the Faculty of Pharmacy and Food Science (University of Barcelona) and kept under controlled conditions of temperature and humidity in a $12 \mathrm{~h}: 12 \mathrm{~h}$ light:dark cycle. This study was conducted in accordance with institutional guidelines for the Care and Use of Laboratory Animals and was approved by the Ethical Committee 
for Animal Experimentation of the University of Barcelona and the Catalonia Government (CEEA/UB ref. 380/13 and DAAM 5988, respectively). The rats were distributed according to diet into three groups ( $\mathrm{n}=12-14$ each one): reference group (RF group) fed standard diet (AIN-93M), cocoa group (CC group) fed a diet containing $10 \%(\mathrm{w} / \mathrm{w})$ cocoa powder (with a content of $2.5 \%$ theobromine), and the theobromine group (TB group) fed a $0.25 \%$ theobromine diet, which had the same amount of theobromine as that provided in the $\mathrm{CC}$ group. Diets containing cocoa and theobromine were prepared in our own laboratory, mixing ingredients (Teklad diets, Envigo, Indianapolis, USA) in the required quantities to provide the same amount of energy, micronutrients and macronutrients as the standard diet (Supporting Table 1). After mixing, food pellets were made and dried. The animals had access to food and water ad libitum throughout the study.

Rats were housed in cages (2-3 rats in each cage). During some periods (days 5 to 7 and days 9 to 14), animals were individually housed in order to establish their motor activity. The nutritional intervention lasted for 7 or 18 days (Figure 1). After these periods, rats were deprived of food for $18 \mathrm{~h}$ and were then euthanized for sample collection.

\section{Data and sample collection}

Food and water intake (FI and WI, respectively) were monitored throughout the study. BW, body length (BL), and rectal body temperature were determined just before euthanasia. Body mass index (BMI), Lee index and food efficiency were also calculated. On days 8 and 19, inguinal and retroperitoneal adipose tissues, heart, liver, right kidney, small intestine and large intestine (colon and caecum) were collected and weighted. Moreover, plasma was obtained and stored at $-80^{\circ} \mathrm{C}$ until processing. At day 8 , inguinal adipose tissue and liver were kept at $-80{ }^{\circ} \mathrm{C}$ until mRNA quantification and/or biochemical variables determination.

\section{Biochemical determinations in plasma and liver}

Plasma total cholesterol, HDL-c, TG, and glucose concentrations were determined by specific colorimetric kits (Química Clínica Aplicada S.A., Spain), following the manufacturer's instructions adapted to a microplate. Absorbance was measured at $505 \mathrm{~nm}$ (microplate Tecan photometer, Tecan 
Group Ltd, Männedorf, Switzerland). LDL cholesterol (LDL-c) concentration was estimated by means of the Friedewald formula $(\mathrm{LDL}-\mathrm{c}=$ total cholesterol $-(\mathrm{HDL}-\mathrm{c}-\mathrm{TG} / 5))$. In order to measure hepatic TG, frozen liver tissue samples were homogenized with a Polytron ${ }^{\circledR}$ (Kinematica, Lucerne, Switzerland) in isopropanol $\left(1 \mathrm{~mL} / 50 \mathrm{mg}\right.$ liver), kept for $1 \mathrm{~h}$ at $4{ }^{\circ} \mathrm{C}$ and then centrifuged $(1313 \mathrm{~g}, 4$ $\left.{ }^{\circ} \mathrm{C}, 5 \mathrm{~min}\right)$. Supernatants were used for TG determination.

\section{Metabolic hormones determination}

Plasma ghrelin, glucagon, glucagon-like peptide (GLP-1) and leptin were evaluated after fasting in 19-day samples, with the Bio-Plex Pro ${ }^{\mathrm{TM}}$ Diabetes Assay (Bio-Rad Laboratories, Madrid, Spain). The lower and upper limits of quantification were: 159.58-155595.25 $\mathrm{pg} / \mathrm{mL}$ (ghrelin), 23.43-1316.37 pg/mL (glucagon), 8.44-1999.39 pg/mL (GLP-1) and 123.73-130750.77 pg/mL (leptin). Plasma insulin was measured using an ultra-sensitive ELISA kit (Ultra Sensitive Rat Insulin ELISA kit) from Crystal Chem (Downers Grove, IL, USA) according to the manufacturer's instructions.

\section{Fecal fat content and $\mathrm{pH}$ determinations}

Feces collected from the whole of day 7 were humidified and submitted to near infrared spectroscopy to determine the fat content by means of an InfraAlyzer 500 (Bran+Luebbe, Norderstedt, Germany). Furthermore, fecal $\mathrm{pH}$ determination was performed in feces from days 7 and 18 using a surface $\mathrm{pH}$ electrode (Crison Instruments, SA, Barcelona, Spain).

\section{Gene expression quantification}

In liver and adipose tissue samples, we determined the gene expression of molecules expressed in particular immune cells, such as CD11b (monocytes, neutrophils, natural killer cells, granulocytes and macrophages), CD11c (monocytes, granulocytes, some B cells, dendritic cells and macrophages), CD68 (monocytes and macrophages), CD4 (predominant on T helper cells, but also in macrophages, monocytes and dendritic cells), $\mathrm{CD} 8 \alpha$ (predominant on cytotoxic $\mathrm{T}$ cells and in natural killer cells and dendritic cells) and Foxp3 (regulatory T cells). In addition, we quantified the gene expression of pro-inflammatory cytokines, such as interleukine (IL)-1 $\beta$, TNF $\alpha$, IL-6, MCP-1 
and IFN $\gamma$, anti-inflammatory cytokines, such as IL-4 and IL-10, and the transcription factors NF- $\mathrm{B}$ (involved in inflammation) and PPAR- $\gamma$ (present in adipose tissue and macrophages and involved in inflammation regulation). For this, liver and adipose tissue samples were homogenized in a lysing matrix tube (MP Biomedicals, Illkirch, France) by a FastPrep-24 equipment (MP Biomedicals, $30 \mathrm{~s}$, at $6.5 \mathrm{~m} / \mathrm{s}$ ). Total RNA was extracted using the RNeasy ${ }^{\circledR}$ mini kit (Qiagen, Madrid, Spain) following the manufacturer's directions. The NanoPhotometer (BioNova Scientific, CA, USA) was used to determine RNA concentration. A thermal cycler PTC-100 Programmable Thermal Controller with TaqMan ${ }^{\circledR}$ Reverse Transcription Reagents (Applied Biosystems, AB, Weiterstadt, Germany) was applied to obtain cDNA. Subsequently, a PCR quantitative assay was carried out (ABI Prism $7900 \mathrm{HT}$, AB) using the following specific PCR TaqMan ${ }^{\circledR}$ primers: CD11b (Rn00709342_m1, Inventoried (I)), CD11c (Rn01511082_m1, I), CD68 (Rn01495634_m1, I), CD4

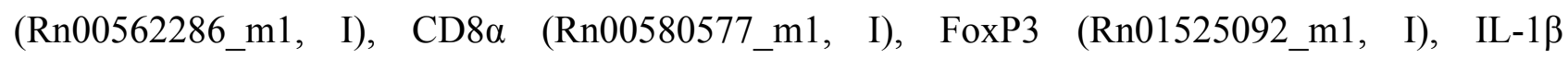
(Rn00580432_m1), IL-10 (Rn00563409_m1, I), IL-6 (Rn01410330_m1, I), TNF $\alpha$

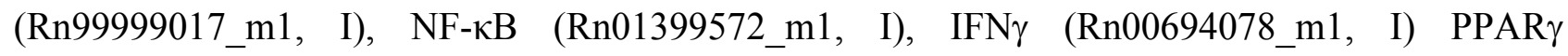
(Rn00440945_m1, I), MCP-1 (Rn00580555_m1, I). The relative amount of target mRNA was normalized using $\beta$-actin (Rn00667869_m1, I) or HPRT1 (Rn01527840_m1, I) as housekeeping genes and the $2^{-\Delta \Delta C t}$ method, as previously described ${ }^{23}$. The gene expression obtained was calculated giving the 1 value to the mean results from the RF group.

\section{Metabolomic analyses}

The untargeted metabolomics analyses were performed on plasma samples collected after 7 days of intervention with a high-performance liquid chromatography coupled to electrospray ionization and quadrupole time-of-flight mass spectrometry (HPLC-ESI-QToF-MS) as previously published ${ }^{24}$. Detailed description is given as Supporting Information (Supporting Table 2).

\section{Motor activity measurement}

Spontaneous motor activity of each animal was continuously recorded on days 5 to 7 and 9 to 14 by means of activity meters placed outside the cage that used two perpendicular crossed infrared beams 
situated $6 \mathrm{~cm}$ above the floor of the cage. Each beam interruption represented an activity count that was registered and stored in 15-min data bins for further analysis. Total daily activity was calculated as well as the nocturnal and diurnal activity separately. Results are expressed as the mean values (counts every 15 minutes).

\section{Statistical analysis}

Data were evaluated with the software package SPSS 22.0 (IBM Statistical Package for the Social Sciences, version 22.0, Chicago, IL, USA) by using the parametric test one-way ANOVA followed by Bonferroni's post hoc test or the nonparametric tests Kruskal-Wallis and Mann-Whitney U in accordance with its homogeneity of variance (Levene test) and distribution (Shapiro-Wilk test). Significant differences were established when $\mathrm{P} \leq 0.05$.

Significant mass features selected after multi- and univariate statistical analyses of the metabolomics dataset were identified by a multistep procedure as specified in Supporting Information. Untargeted metabolomics data were analyzed by multivariate statistical methods using SIMCA-P+ 13.0 software (Umetrics, Umea, Sweden). Particular statistical analysis for these data is expanded in the Supporting Information.

The metabolites identified by the untargeted metabolomics analysis were correlated with the results from BW gain, BMI, Lee index, FI, WI, inguinal, retroperitoneal, small and large intestine tissue weights, fecal fat, plasma total cholesterol, HDL-c and LDL-c concentrations and hepatic TG levels. For this, after a log-transformation of results, Spearman's correlation analyses were performed. The Benjamini-Hochberg method was used to adjust p-values for multiple testing considering a 5\% false discovery rate.

\section{RESULTS}

\section{Morphometric variables}

Morphometric changes were already detected after 7 days of the experimental diets (Table 1). Specifically, the animals from the CC and TB groups had lower BW gain $(\mathrm{P}<0.001)$. Moreover, the 
rats fed $\mathrm{CC}$ and $\mathrm{TB}$ diets for 18 days presented lower body length than those of the RF group $(\mathrm{P}<0.001)$. Decreased BMI was found in the $\mathrm{CC}$ and TB groups $(\mathrm{P}<0.05)$ on days 8 and 19 and, additionally, on day 8 the Lee index in both interventional groups was lower than in the RF animals $(\mathrm{P}<0.05)$. In any case, no differences were detected between $\mathrm{CC}$ and TB groups, the changes being almost identical between them.

In spite of the lower values in morphometric changes, mean FI did not decrease in the CC or TB groups, but mean WI increased in both interventional groups compared to the RF one $(\mathrm{P}<0.001)$ (Table 1). With regard to food efficiency, the CC and TB groups showed lower values than the RF animals, which was already evidenced after one single week of $\operatorname{diet}(\mathrm{P}<0.05)$ (Table 1).

\section{Body temperature and spontaneous physical activity}

Rectal temperature was measured at days 7 and 18. There were no changes in body temperature between the considered groups at any of the studied time points (Table 1). Moreover, motor activity was individually measured between days 5 to 7 and days 9 to 14. In the first period, cocoa diet decreased diurnal motor activity compared to the RF group (Figure 2a-b) without statistically significant changes when considering the whole day. In the second period, higher activity was observed in animals fed cocoa diet than in those from the RF group, both in diurnal and nocturnal determinations. The activity in the TB group did not achieve a significant modification compared to RF animals (Figure 2c- $\mathbf{d})$.

\section{Relative organ and tissue weights}

After euthanasia on days 8 or 19, some organs and adipose tissues were collected and weighed (Table 1). Animals from the $\mathrm{CC}$ and $\mathrm{TB}$ groups showed lower relative retroperitoneal fat weight at both time points $(\mathrm{P}<0.05)$, although the relative inguinal fat weight was lower only in the TB group after 18 days of diet. The rats fed cocoa for 18 days had higher relative heart weight $(\mathrm{P}<0.05)$. In the two studied time points no changes in the relative kidney weight were observed. However, the CC and TB groups had lower relative liver weight than the RF group $(\mathrm{P}<0.01)$, the TB value being even lower than that of the CC group after 18 days of diet $(\mathrm{P}<0.05)$. On the other hand, the relative 
small and large intestinal weights were higher after 7 and 18 days of the two experimental diets in comparison to the RF group $(\mathrm{P}<0.05)$ (Table 1).

\section{Fecal fat and pH values}

Fecal fat content, determined after 7 days of diet, was lower in cocoa- and theobromine-fed animals in comparison to the RF group $(\mathrm{P}<0.01)$, and even the rats from the $\mathrm{CC}$ group showed less fecal fat levels than the TB group $(\mathrm{P}<0.05)$ (Figure 3). Otherwise, cocoa diet lowered fecal $\mathrm{pH}$ detected at day 18 in comparison to the RF and TB groups $(\mathrm{P}<0.05)$ (Figure 3).

\section{Cholesterol and triglyceride concentrations}

After 7 days of diet, the total plasma cholesterol concentrations in both $\mathrm{CC}$ and TB groups were about $15 \%$ lower than those detected in the RF group $(\mathrm{P}<0.05)$ (Table 2). Nevertheless, no differences were detected later. Similar results were observed in LDL-c content. Interestingly, HDL-c values increased by $24-41 \%$ in the $\mathrm{CC}$ and TB groups at both studied time points $(\mathrm{P}<0.05)$ (Table 2). Moreover, plasma TG concentrations from the TB group were significantly lower after 18 days of diet $(\mathrm{P}<0.01)$ (Table 2) while hepatic TG concentrations decreased after both cocoa and theobromine diets, this already observable just after one week of $\operatorname{diet}(\mathrm{P}<0.001)$ (Table 2).

\section{Liver and adipose tissue gene expression}

The influence of diets containing cocoa or theobromine on the gene expression of some immune molecules in hepatic and adipose tissues was quantified after one week of diet (Figures 4 and 5). With regard to the liver (Figure 4), cocoa-enriched diet reduced the gene expression of CD11c and CD4 without inducing significant changes in CD11b, CD68, Foxp3, TNF- $\alpha$, NF- $\kappa$ B and PPAR- $\gamma$. Moreover, both CC and TB groups showed lower mRNA levels of CD8 $\alpha$, IL-10 and IL-1 $\beta$ with respect to the RF group. The TB group had higher TNF- $\alpha$ and NF- $\kappa \mathrm{B}$ hepatic gene expressions than the RF and $\mathrm{CC}$ groups $(\mathrm{P}<0.05)$. In this tissue, the mRNA levels of IL-4, IL-6, MCP-1 were undetectable in any of the considered groups.

With regard to adipose tissue (Figure 5), the gene expression of CD11b, CD11c, Foxp3, IFN- $\gamma$, TNF- $\alpha$, IL-4, and IL-6 was undetectable. Both nutritional interventions induced an upregulation of 
the CD68 and MCP-1 gene expression and a downregulation of the CD4 expression with respect to the RF group $(\mathrm{P}<0.05)$. Moreover, animals fed cocoa had higher gene expression of IL-10 and IL-1 $\beta$ whereas the TB group showed lower gene expression of CD8 $\alpha$ in comparison to the RF animals. No changes in NF- $\mathrm{BB}$ and PPAR- $\gamma$ expressions were found in the $\mathrm{CC}$ and TB groups.

\section{Glucose metabolism}

After 7 days of diet, the plasma glucose concentrations in fasting conditions were similar within groups, however, after 18 days, the CC and TB groups had higher plasma glucose concentrations than the RF group (Table 2). At this later time point, plasma ghrelin, glucagon, GLP-1, leptin and insulin concentrations were determined (Figure 6). The rats from $\mathrm{CC}$ and $\mathrm{TB}$ groups showed higher ghrelin concentrations and lower glucagon levels than the RF group $(\mathrm{P}<0.01$ and $\mathrm{P}<0.05$, respectively). In fasting conditions, GLP-1 and leptin levels were below the limit of detection in the three experimental groups. There was no difference in plasma insulin levels among the studied groups, although a tendency to decrease $(\mathrm{P}=0.092)$ was observed after both experimental diets.

\section{Metabolomic analyses}

The metabolomic analyses are detailed in the supporting information (Supporting Information Figure S1 and Tables S3 and S4). As mentioned in the Material and Methods section, only features showing high correlation coefficients $(\mid p($ corr $) \mid>0.75)$ in all models developed during the leaveone-out procedure were included in the list of discriminating metabolites explaining the differences between groups, which were then submitted to the metabolite identification procedure. A total of 185 features fulfilled this criteria, 135 features from the ESI $(+)$ data set and 50 features from the ESI(-) data set. The scores plot of the three-group PLS-DA models with non-OSC filtered data using the selected features revealed higher differences (displayed through PC1) between the REF group and the other two groups, followed by differences between the animals supplemented with cocoa and those supplemented with theobromine (PC2), indicating that each diet induced a different metabolomic pattern (Figure 7). Among the features selected as significant, eight of them were assigned to the six compounds that were identified as discriminant metabolites. They were related 
to at least one of the study groups: theobromine, pantothenic acid, glycocholic acid, citrulline, 5-(2'carboxyethyl)-4,6-dihydroxypicolinate (CEDHP), and 5-hydroxyindoleacetic acid (HIAA) (Figure 8). Mass features of these compounds are listed in the Supporting Information Table S5. Theobromine was an exogenous metabolite only found in the CC and TB groups and, as expected, there were no differences between the two experimental diets. As endogenous metabolites, the CC and TB groups showed higher concentrations of pantothenic acid and glycocholic acid than the RF group ( $\mathrm{p}<0.05)$. Moreover, the TB group exhibited higher levels of citrulline compared with the RF group, and higher levels of CEDHP in comparison to both the RF and $\mathrm{CC}$ groups $(\mathrm{P}<0.05)$. Finally, the intake of both cocoa- and theobromine-enriched diets induced lower levels of HIAA than those in the RF group.

\section{Correlations between morphometric and biochemical data, and the discriminant metabolites}

In order to determine the relationship between the discriminant metabolites and morphometric and biochemical significant results obtained at the same time point, correlations between both data were carried out (Figure 9). Theobromine plasma concentration correlated negatively with BMI, retroperitoneal fat weight and hepatic TG, whereas it correlated positively with WI, small and large intestine weights as well as with plasma HDL-c levels. Almost similarly, the plasma concentrations of pantothenic acid negatively correlated with BW gain, BMI, food efficiency and hepatic TG while it correlated directly with higher WI and small intestine weight.

Moreover, the more plasma concentration of glycocholic acid detected, the lower the fecal fat observed. Likewise, higher citrulline concentrations correlated with higher levels of HDL-c. The amount of the metabolite CEDHP correlated positively with the large intestinal relative weight and negatively with plasma TG. Regarding the metabolite HIAA, found in lower concentrations in the $\mathrm{CC}$ and $\mathrm{TB}$ groups, it positively correlated with lower BW gain, BMI, fecal fat content, total cholesterol, LDL-c and plasma TG. Otherwise, HIAA correlated negatively with WI and small and large intestinal relative weights. 
Regarding the Lee index, FI and inguinal fat weight, no significant correlation was detected with any of the studied metabolites.

\section{DISCUSSION}

Previous studies have reported the effect of cocoa on metabolism ${ }^{8,11-13,25,26}$. However, the exact compound responsible for such effect and the length needed to achieve such benefits still remain unknown and are a topic of debate. Although polyphenols and methylxanthines are recognized bioactive compounds in $\operatorname{cocoa}^{3}$, a vast number of studies have associated the cocoa role in metabolism only with its polyphenol content. In the current study, we evidence the importance of theobromine in the metabolic properties of cocoa as well as its rapid effects.

Rats fed with $10 \%$ cocoa that consumed a similar or even higher amount of food than the RF group, underwent a lower body weight gain as early as after 7 days of diet, which was accompanied by lower BMI and Lee's index. Importantly, theobromine on its own produced similar actions. Cocoa effects on body weight increase have already been reported both in animal models and humans ${ }^{11-}$ $13,15,27$ and it has been postulated that cocoa is a weight loss accelerator ${ }^{28}$. Our results concerning body temperature and motor activity demonstrated that, at least after the first week of diet, there was no increase in the metabolic rate. The metabolomic analyses carried out after 7 days of diet showed that BMI inversely correlated with the plasma concentrations of theobromine, which is in line with a previous study showing a negative association of BMI with the amount of theobromine in urine of rats fed cocoa for 3 weeks ${ }^{29}$. On the other hand, we found a higher water intake in animals fed cocoa or theobromine, data that correlated directly with the plasma concentrations of theobromine. This higher water intake could be a consequence of the theobromine's diuretic actions $^{30}$ in order to compensate the high volume of excreted urine. Overall, these results allow us to conclude that cocoa theobromine content is an important cocoa metabolite involved in the body weight homeostasis. 
In line with a reduced body weight gain and BMI, rats fed cocoa or theobromine diets had a lower amount of retroperitoneal fat after 7 and 18 days of diet. These results agree with those reported in high-fat-fed obese mice fed $8 \%$ cocoa powder ${ }^{11}$ and with a study carried out in European adolescents reporting that higher chocolate consumption was related with lower fatness ${ }^{31}$. After 7 days of diet, as observed by correlations after metabolomic analyses, the higher the plasma theobromine concentration, the lower the amount of retroperitoneal fat found. Thus, it can be suggested that these cocoa effects on adipose tissue could be due to its theobromine content. However, the proportion of inguinal fat in the theobromine group at day 19 was lower than that in the cocoa group, suggesting that other cocoa compounds (polyphenols and/or fiber) could attenuate this particular effect. On the other hand, adipose tissue is an immune organ that links metabolism and immunity ${ }^{32}$. In this sense, it has been described that cocoa intake decreased macrophage infiltration in adipose tissue in obese mice ${ }^{11}$. However, in this tissue, we have detected a higher gene expression of CD68 and MCP-1 (molecules present in macrophages and involved in their recruitment, respectively) after cocoa and theobromine intake, and higher IL-1 $\beta$ gene expression in cocoa-fed rats. These discrepancies could be due to the status of the animals - healthy status in the current study and obese status in the first described case ${ }^{11}$ - which had already shown an increased macrophage infiltration before cocoa intake. Nevertheless, although some macrophage-related molecules increased, other transcriptional factors related to macrophage or immune activation, such as NF- $\mathrm{BB}$ and PPAR- $\gamma$, were not modified by cocoa or theobromine diets, and, interestingly, the cocoa diet increased the adipocyte gene expression of the anti-inflammatory cytokine IL- 10 . Similarly, the gene expression of CD4 was down-regulated by cocoa and theobromine intake, and that of CD8 only by the theobromine diet. Both CD4 and CD8 are mainly related to lymphocytes and their decrease suggests that there was lower lymphocyte infiltration into the fat. In short, results of adipocyte gene expression suggest that cocoa, partially due to its theobromine content, may be involved in avoiding the low-grade inflammatory status observed in obesity ${ }^{32}$. Nevertheless, because the effect in fat gene expression of both cocoa- and theobromine-enriched diets differed in 
some molecules, the role of other cocoa bioactive compounds such as polyphenols or fiber cannot be discarded.

Fecal samples obtained after 7 days of cocoa or theobromine diet also provided significant results regarding fat content. We found that the fecal fat content in animals fed theobromine and cocoa decreased. These results do not agree with those reported in obese mice fed a cocoa diet ${ }^{11}$ or in obese animals fed with other phytotherapeutic diets ${ }^{33}$. This disagreement could again be due to the different status of the animals or to the different technique used. Nevertheless, we observed that the two experimental diets exerted a similar effect, although it was more obvious in the cocoa group, suggesting the role of other compounds present in the whole cocoa diet in fat excretion.

A relevant outcome observed in the current study was the influence of cocoa and theobromine on plasma lipids such as cholesterol. Although we found that cocoa diet decreased plasma concentrations of total cholesterol and LDL-c, after 7 days of diet, this effect was not longer detected. In fact, it has been reported that in rats fed a high-fat diet with $12.5 \%$ cocoa for 21 days ${ }^{34}$ no decrease was seen in either plasma cholesterol or LDL-c levels, which also agree with studies carried out in humans ${ }^{35,36}$. Nevertheless, both the cocoa diet and the diet containing only theobromine increased plasma HDL-c levels. Similar effects have been reported in humans after a nutritional intervention with $\operatorname{cocoa}^{35,36}$ and with rats fed a high-fat diet plus cocoa ${ }^{34}$. The increase in HDL-c concentration correlated with the plasma levels of theobromine. In partial agreement with this, a recent study in humans taking $500 \mathrm{mg}$ /day theobromine for 4 weeks ${ }^{37}$ demonstrates that theobromine consumption tended to increase fasting plasma HDL-c concentrations. Overall, the current results indicate that in the reported beneficial effects of cocoa intake on plasma lipids, theobromine from cocoa content plays a relevant role. Although the results obtained from the cocoa diet agree with a previous study showing a downregulation of colonic Adipoq gene expression, which is involved in lipid metabolism ${ }^{38}$, further studies must be carried out to establish the effects of both cocoa and theobromine on key enzymes related to lipid metabolism. 
On the other hand, cocoa diet and its methylxanthine alone influence the hepatic tissue. After one week of both diets there was a reduction in hepatic triglycerides and changes in the gene expression of several molecules. These results on hepatic triglycerides are in line with those reported in adult mice fed with a high-fat diet plus $8 \% \operatorname{cocoa}^{11}$, although the effects obtained here were more obvious, which could be due to the use of younger animals fed a standard diet, the higher cocoa proportion and/or the cocoa's composition. Anyway, despite the fact that the effect of cocoa in the suppression of hepatic fatty acid synthesis and transport systems in mesenteric white adipose tissue has been reported ${ }^{34}$, the influence of cocoa on hepatic triglycerides deserves a more in-depth study. We also studied the hepatic gene expression of molecules involved in promoting or inhibiting inflammation. Although both diets decreased the gene expression of the pro-inflammatory cytokine IL-1 $\beta$ and cocoa intake down-regulated CD11c expression, there was a decrease in the expression of the anti-inflammatory IL-10 and theobromine intake increased the gene expression of TNF- $\alpha$ and NF- $\kappa \mathrm{B}$, promoting inflammation. On the other hand, CD4 and CD8 molecule gene expressions were down-regulated by cocoa and/or theobromine, suggesting lower lymphocyte presence in the liver. Overall, the results of the hepatic genes studied do not clarify whether immune mechanisms were involved in the effects of cocoa on the liver. Moreover, the intake of cocoa and theobromine did not show identical effects on the molecules considered, indicating that other bioactive compounds included in the whole cocoa diet can enhance or attenuate that of theobromine alone.

As previous studies demonstrated the influence of a cocoa diet on glucose metabolism ${ }^{12}$, we also tested plasma glucose concentrations, which did not vary after 7 days of diet but increased in the 18-day samples in a similar way in both cocoa- and theobromine-fed animals. Therefore, we determined insulin concentrations, as well as hormones associated with glucose metabolism at this time point. The current study revealed that cocoa and theobromine on its own increased ghrelin levels and decreased glucagon concentration, as already reported in rats fed $10 \% \operatorname{cocoa}^{29}$. Ghrelin, also known as the hunger hormone, is a multifaceted hormone secreted by the stomach, which is influenced by the BMI, glucose and insulin levels, among other factors ${ }^{39}$. Ghrelin is involved in the 
inhibition of insulin secretion and contributes to glucose homeostasis, apart from regulating energy homeostasis ${ }^{40}$. Otherwise, glucagon is a hormone produced in the $\alpha$ cells of the pancreatic islets stimulated by low levels of glucose ${ }^{41}$. From our results obtained in fasting samples after 18 days of cocoa or theobromine diets, we can suggest that the higher ghrelin secretion might be due to stimulation from the lower BMI and could increase plasma glucose concentration, whereas it decreased glucagon levels and tended to decrease plasma insulin. The discrepancy between the current results and previous studies that show the antidiabetic effects of a cocoa diet could be due to the age of the rats at the beginning of the nutritional intervention as well as the use of healthy rather than diabetic animals. In fact, a study in humans taking dark chocolate found decreased plasma glucose concentrations when $\mathrm{BMI} \geq 25 \mathrm{~kg} / \mathrm{m}^{2}$ but no changes when $\mathrm{BMI}<25 \mathrm{~kg} / \mathrm{m}^{2}$ 42. Although further studies are required to check the importance of age and the existence of diabetes/overweight in the cocoa/theobromine effects, our findings suggest that theobromine plays an important role in the influence of cocoa on glucose metabolism. In this sense, it has been reported that cocoa intake produces a downregulation of colonic Adipoq gene expression involved in glucose metabolism as well as that of lipids, as has been mentioned above $\mathrm{e}^{38}$.

Finally, it is important to highlight the levels of endogenous discriminant metabolites found after cocoa and/or theobromine intake and their correlations with some outcomes obtained after 7 days of diet. Higher plasma pantothenic acid was correlated with lower body weight gain, BMI, food efficiency and hepatic triglycerides, and higher water intake and small intestine weight. The pantothenic acid (vitamin B5) is a precursor for the coenzyme A (CoA) and acyl carrier protein, involved in the metabolism of steroids, fatty acids and phosphatides ${ }^{43}$. CoA is found in the pathway of cholesterol synthesis, amino acid catabolism, and is a crucial substrate in the oxidation of fatty acids $^{44}$. The higher levels of pantothenic acid in cocoa and theobromine groups might be associated with an enhanced fatty-acid metabolism that would partially explain the lower body weight gain and the decrease in hepatic triglycerides as well as plasma cholesterol levels. Also related to the lipid metabolism, we found a negative correlation between the elevated glycocholic acid levels in cocoa 
and theobromine groups and their reduced fecal fat content. As glycocholic acid is an indirect cholesterol-derived bile acid ${ }^{45}$, it can be suggested that a high amount of glycocholic in plasma could promote lipid metabolism.

On the other hand, plasma citrulline concentrations were directly associated with those of HDL-c. Citrulline is a nonessential amino acid, precursor of arginine. In particular, from arginine and different cofactors NO-synthase produced NO with release of citrulline ${ }^{46}$. Although with the current results we cannot evidence the exact pathway affected by the citrulline increase, it has been reported, in agreement with the current results, that the supplementation of L-citrulline is associated with increased HDL-c levels ${ }^{47}$.

The metabolite 5-(2'-carboxyethyl)-4,6-dihydroxypicolinate (CEDHP) was found in higher plasma concentrations in those animals receiving theobromine. This compound was derived from the tryptophan metabolism and comes from the kynurenine pathway. Our results are in line with the increased kynurenine production observed after black tea consumption ${ }^{48}$. In particular, CEDHP levels inversely correlated with plasma triglycerides, suggesting the involvement of this pathway, which is beneficial for the triglyceridemia status. Another metabolite indicating the implication of this pathway is the HIAA, which is the only one that was found in lower concentrations after cocoa and theobromine intake in comparison with the animals fed standard diet. HIAA was associated with a lot of changes found after cocoa or theobromine consumption, such as body weight increase, water intake, small and large intestine weights, fecal fat content and with plasma cholesterol and hepatic triglyceride levels. HIAA is involved in the tryptophan metabolism system, as it is a breakdown product of serotonin and considered as a marker for the endogenous serotonin turnover $^{49}$. In fact, higher urinary HIAA excretion in rats has been reported after cocoa intake and has been related to cocoa tryptophan degradation by gut microbiota ${ }^{29}$. Therefore, it can be suggested that the changes in the microbiota induced by cocoa and theobromine would reduce the absorption of tryptophan that will be found in lower plasma concentrations after one week of diets containing both products. Interestingly, such lower absorption seems related to lower plasma cholesterol and 
441 triglyceride concentrations. In this line, it has been shown that severe tryptophan restriction 442 decreased body weight, body fat and lean mass in obese-prone rats ${ }^{50}$.

443 In conclusion, from the results obtained in rats fed cocoa or only theobromine, we can conclude that 444 this methylxanthine is the main factor responsible for cocoa's effects on body weight gain as well as 445 on the lipid and glucose metabolism. These effects on body weight and lipids appeared as early as 446 after 7 days of diet, whereas those affecting glucose metabolism require a longer intervention. 
447

448

449

450

451

452

453

454

455

456

457

458

459

460

461

462

\section{Abbreviations}

AB, Applied Biosystems; BL, body length; BMI, body mass index; BW, body weight; CC, cocoa group; CEDHP, 5-(2’-carboxyethyl)-4,6-dihydroxypicolinate; CoA, coenzyme A; FI, food intake; GLP-1, glucagon-like peptide; HDL-c, high-density lipoprotein cholesterol; HIAA, hydroxyindoleacetic acid; HPLC-ESI-QToF-MS, high-performance liquid chromatography coupled to electrospray ionization and quadrupole time-of-flight mass spectrometry; IL, interleukin; LDL-c, low-density lipoprotein cholesterol; OSC-PLS-DA, partial least squares discriminant analysis with orthogonal signal correction; PCA, principal components analyses; PCR, polymerase chain reaction; QC, quality control; RF, reference group; TB; theobromine group; TG, triglycerides; WI, water intake

\section{Acknowledgments}

The authors would like to thank Idilia Foods S.L. for providing the cocoa powder. RGD thanks the "Juan de la Cierva" program from MINECO (FJCI-2015-26590). The authors would like to thank Marc Bitlloch and Sílvia Plana for their help in the laboratory work.

\section{Funding sources}

This study was supported by a grant from INSA-UB (FRI-2014) and from the Spanish Ministry of Economy and Competitiveness (AGL2011-24279). MGA, RGD and CAL also thanks the SGR2017-1556 from the Generalitat de Catalunya's Agency AGAUR.

\section{References}

(1) Katz, D. L.; Doughty, K.; Ali, A. Cocoa and Chocolate in Human Health and Disease. Antioxid. Redox Signal. 2011, 15, 2779-2811.

(2) Di Mattia, C. D.; Sacchetti, G.; Mastrocola, D.; Serafini, M. From Cocoa to Chocolate: The 
Impact of Processing on in Vitro Antioxidant Activity and the Effects of Chocolate on Antioxidant Markers in Vivo. Front. Immunol. 2017, 8, 1207.

471

472

473

(3) Franco, R.; Oñatibia-Astibia, A.; Martínez-Pinilla, E. Health Benefits of Methylxanthines in Cacao and Chocolate. Nutrients 2013, 5, 4159-4173.

(4) Magrone, T.; Russo, M. A.; Jirillo, E. Cocoa and Dark Chocolate Polyphenols: From Biology to Clinical Applications. Front. Immunol. 2017, 8, 1-13.

(5) Camps-Bossacoma, M.; Massot-Cladera, M.; Abril-Gil, M.; Franch, A.; Pérez-Cano, F. J.; Castell, M. Cocoa Diet and Antibody Immune Response in Preclinical Studies. Front. Nutr. 2017, 4, 1-14.

(6) Camps-Bossacoma, M.; Pérez-Cano, F. J.; Franch, À.; Castell, M. Gut Microbiota in a Rat Oral Sensitization Model: Effect of a Cocoa-Enriched Diet. Oxid. Med. Cell. Longev. 2017, 2017, 1-12.

(7) Pérez-Cano, F. J.; Massot-Cladera, M.; Franch, A.; Castellote, C.; Castell, M. The Effects of Cocoa on the Immune System. Front. Pharmacol. 2013, 4, 71.

(8) Ellam, S.; Williamson, G. Cocoa and Human Health. Annu. Rev. Nutr. 2013, 33, 105-128.

(9) Loffredo, L.; Perri, L.; Nocella, C.; Violi, F. Antioxidant and Antiplatelet Activity by Polyphenol-Rich Nutrients: Focus on Extra Virgin Olive Oil and Cocoa. Br. J. Clin.

Pharmacol. 2017, 83, 96-102.

(10) Grassi, D.; Desideri, G.; Mai, F.; Martella, L.; De Feo, M.; Soddu, D.; Fellini, E.; Veneri, M.; Stamerra, C. A.; Ferri, C. Cocoa, Glucose Tolerance, and Insulin Signaling: Cardiometabolic Protection. J. Agric. Food Chem. 2015, 63, 9919-9926.

(11) Gu, Y.; Yu, S.; Lambert, J. D. Dietary Cocoa Ameliorates Obesity-Related Inflammation in High Fat-Fed Mice. Eur. J. Nutr. 2014, 53, 149-158.

(12) Cordero-Herrera, I.; Martín, M. Á.; Escrivá, F.; Álvarez, C.; Goya, L.; Ramos, S. Cocoa-Rich Diet Ameliorates Hepatic Insulin Resistance by Modulating Insulin Signaling and Glucose Homeostasis in Zucker Diabetic Fatty Rats. J. Nutr. Biochem. 2015, 26, 704-712. 
(13) Min, S. Y.; Yang, H.; Seo, S. G.; Shin, S. H.; Chung, M. Y.; Kim, J.; Lee, S. J.; Lee, H. J.; Lee, K. W. Cocoa Polyphenols Suppress Adipogenesis in Vitro and Obesity in Vivo by Targeting Insulin Receptor. Int. J. Obes. 2013, 37, 584-592.

(14) Jalil, A. M. M.; Ismail, A.; Pei, P. C.; Hamid, M.; Kamaruddin, S. H. S. Effects of Cocoa Extract Containing Polyphenols and Methylxanthines on Biochemical Parameters of ObeseDiabetic Rats. J. Sci. Food Agric. 2009, 89, 130-137.

(15) Nickols-Richardson, S. M.; Piehowski, K. E.; Metzgar, C. J.; Miller, D. L.; Preston, A. G. Changes in Body Weight, Blood Pressure and Selected Metabolic Biomarkers with an Energy-Restricted Diet Including Twice Daily Sweet Snacks and Once Daily Sugar-Free Beverage. Nutr. Res. Pract. 2014, 8, 695-704.

(16) Renzo, L. D. I.; Rizzo, M.; Colica, C.; Iacopino, L.; Domino, E.; Sergi, D.; Lorenzo, A. D. E. Effects of Dark Chocolate in a Population of Normal Weight Obese Women: A Pilot Study. Eur. Rev. Med. Pharmacol. Sci. 2013, 17, 2257-2266.

(17) Martínez-López, S.; Sarriá, B.; Sierra-Cinos, J. L.; Goya, L.; Mateos, R.; Bravo, L. Realistic Intake of a Flavanol-Rich Soluble Cocoa Product Increases HDL-Cholesterol without Inducing Anthropometric Changes in Healthy and Moderately Hypercholesterolemic Subjects. Food Funct. 2014, 5, 364.

(18) Massot-Cladera, M.; Costabile, A.; Childs, C. E.; Yaqoob, P.; Franch, À.; Castell, M.; PérezCano, F. J. Prebiotic Effects of Cocoa Fibre on Rats. J. Funct. Foods 2015, 19, 341-352.

(19) Ramiro-Puig, E.; Pérez-Cano, F. J.; Ramos-Romero, S.; Pérez-Berezo, T.; Castellote, C.; Permanyer, J.; Franch, A.; Izquierdo-Pulido, M.; Castell, M. Intestinal Immune System of Young Rats Influenced by Cocoa-Enriched Diet. J. Nutr. Biochem. 2008, 19, 555-565.

(20) Pérez-Berezo, T.; Franch, A.; Ramos-Romero, S.; Castellote, C.; Pérez-Cano, F. J.; Castell, M. Cocoa-Enriched Diets Modulate Intestinal and Systemic Humoral Immune Response in Young Adult Rats. Mol. Nutr. Food Res. 2011, 55 Suppl 1, S56-66.

(21) Camps-Bossacoma, M.; Abril-Gil, M.; Saldaña-Ruiz, S.; Franch, À.; Pérez-Cano, F. J.; 
Castell, M. Cocoa Diet Prevents Antibody Synthesis and Modifies Lymph Node Composition and Functionality in a Rat Oral Sensitization Model. Nutrients 2016, 8, 242.

(22) Camps-Bossacoma, M.; Pérez-Cano, F. J.; Franch, À.; Castell, M. Theobromine Is Responsible for the Effects of Cocoa on the Antibody Immune Status of Rats. J. Nutr. 2018, $148,646-671$.

(23) Camps-Bossacoma, M.; Abril-Gil, M.; Franch, À.; Pérez-Cano, F. J.; Castell, M. Induction of An Oral Sensitization Model in Rats. Clin. Immunol. Endocr. Metab. Drugs 2014, 1, 89-101.

(24) Tulipani, S.; Mora-Cubillos, X.; Jáuregui, O.; Llorach, R.; García-Fuentes, E.; Tinahones, F. J.; Andres-Lacueva, C. New and Vintage Solutions to Enhance the Plasma Metabolome Coverage by LC-ESI-MS Untargeted Metabolomics: The Not-so-Simple Process of Method Performance Evaluation. Anal. Chem. 2015, 87, 2639-2647.

(25) Latif, R. Health Benefits of Cocoa. Curr. Opin. Clin. Nutr. Metab. Care 2013, 16, 669-674.

(26) Strat, K. M.; Rowley, T. J.; Smithson, A. T.; Tessem, J. S.; Hulver, M. W.; Liu, D.; Davy, B. M.; Davy, K. P.; Neilson, A. P. Mechanisms by Which Cocoa Flavanols Improve Metabolic Syndrome and Related Disorders. J. Nutr. Biochem. 2016, 35, 1-21.

(27) Rodríguez-Lagunas, M. J.; Vicente, F.; Pereira, P.; Castell, M.; Pérez-Cano, F. J.

Relationship between Cocoa Intake and Healthy Status: A Pilot Study in University Students. Molecules 2019, 24, 812.

(28) Bohannon, J.; Koch, D.; Homm, P.; Driehaus, A. Chocolate with High Cocoa Content as a Weight-Loss Accelerator. Int. Arch. Med. 2015, 8, 1-8.

(29) Massot-Cladera, M.; Mayneris-Perxachs, J.; Costabile, A.; Swann, J. R.; Franch, À.; PérezCano, F. J.; Castell, M. Association between Urinary Metabolic Profile and the Intestinal Effects of Cocoa in Rats. Br. J. Nutr. 2017, 117, 623-634.

(30) Osswald, H.; Schnermann, J. Methylxanthines and the Kidney. Handb. Exp. Pharmacol. 2011, 200, 391-412.

(31) Cuenca-García, M.; Ruiz, J. R.; Ortega, F. B.; Castillo, M. J. Association between Chocolate 
Consumption and Fatness in European Adolescents. Nutrition 2014, 30, 236-239.

(32) Stolarczyk, E. Adipose Tissue Inflammation in Obesity: A Metabolic or Immune Response? Curr. Opin. Pharmacol. 2017, 37, 35-40.

(33) Kim, S.; Lee, M.-S.; Jung, S.; Son, H.-Y.; Park, S.; Kang, B.; Kim, S.-Y.; Kim, I.-H.; Kim, C.-T.; Kim, Y. Ginger Extract Ameliorates Obesity and Inflammation via Regulating MicroRNA-21/132 Expression and AMPK Activation in White Adipose Tissue. Nutrients 2018, 10, 1567.

(34) Matsui, N.; Ito, R.; Nishimura, E.; Yoshikawa, M.; Kato, M.; Kamei, M.; Shibata, H.; Matsumoto, I.; Abe, K.; Hashizume, S. Ingested Cocoa Can Prevent High-Fat Diet-Induced Obesity by Regulating the Expression of Genes for Fatty Acid Metabolism. Nutrition 2005, $21,594-601$.

(35) Khan, N.; Monagas, M.; Andres-Lacueva, C.; Casas, R.; Urpí-Sardà, M.; Lamuela-Raventós, R. M.; Estruch, R. Regular Consumption of Cocoa Powder with Milk Increases HDL Cholesterol and Reduces Oxidized LDL Levels in Subjects at High-Risk of Cardiovascular Disease. Nutr. Metab. Cardiovasc. Dis. 2012, 22, 1046-1053.

(36) Baba, S.; Natsume, M.; Yasuda, A.; Nakamura, Y.; Tamura, T.; Osakabe, N.; Kanegae, M.; Kondo, K. Plasma LDL and HDL Cholesterol and Oxidized LDL Concentrations Are Altered in Normo- and Hypercholesterolemic Humans after Intake of Different Levels of Cocoa Powder. J. Nutr. 2007, 137, 1436-1441.

(37) Smolders, L.; Mensink, R. P.; Boekschoten, M. V.; de Ridder, R. J. J.; Plat, J. Theobromine Does Not Affect Postprandial Lipid Metabolism and Duodenal Gene Expression, but Has Unfavorable Effects on Postprandial Glucose and Insulin Responses in Humans. Clin. Nutr. 2018, 37, 719-727.

(38) Massot-Cladera, M.; Franch, À.; Castell, M.; Pérez-Cano, F. J. Cocoa Polyphenols and Fiber Modify Colonic Gene Expression in Rats. Eur. J. Nutr. 2017, 56, 1871-1885.

(39) Klok, M. D.; Jakobsdottir, S.; Drent, M. L. The Role of Leptin and Ghrelin in the Regulation 
of Food Intake and Body Weight in Humans: A Review. Obes. Rev. 2007, 8, 21-34.

574

575

576

(40) Pradhan, G.; Samson, S. L.; Sun, Y. Ghrelin: Much More than a Hunger Hormone. Curr. Opin. Clin. Nutr. Metab. Care 2013, 16, 619-624.

(41) Habegger, K. M.; Heppner, K. M.; Geary, N.; Bartness, T. J.; DiMarchi, R.; Tschöp, M. H. The Metabolic Actions of Glucagon Revisited. Nat. Rev. Endocrinol. 2010, 6, 689-697.

(42) Almoosawi, S.; Tsang, C.; Ostertag, L. M.; Fyfe, L.; Al-Dujaili, E. A. S. Differential Effect of Polyphenol-Rich Dark Chocolate on Biomarkers of Glucose Metabolism and Cardiovascular Risk Factors in Healthy, Overweight and Obese Subjects: A Randomized Clinical Trial. Food Funct. 2012, 3, 1035-1043.

(43) Rychlik, M. Pantothenic Acid Quantification by a Stable Isotope Dilution Assay Based on Liquid Chromatography-Tandem Mass Spectrometry. Analyst 2003, 128, 832-837.

(44) Boaz, S. M.; Champagne, C. D.; Fowler, M. A.; Houser, D. H.; Crocker, D. E. Water-Soluble Vitamin Homeostasis in Fasting Northern Elephant Seals (Mirounga Angustirostris) Measured by Metabolomics Analysis and Standard Methods. Comp. Biochem. Physiol. - A Mol. Integr. Physiol. 2012, 161, 114-121.

(45) Zhang, A.; Sun, H.; Yan, G.; Han, Y.; Ye, Y.; Wang, X. Urinary Metabolic Profiling Identifies a Key Role for Glycocholic Acid in Human Liver Cancer by Ultra-Performance Liquid-Chromatography Coupled with High-Definition Mass Spectrometry. Clin. Chim. Acta 2013, 418, 86-90.

(46) Curis, E.; Nicolis, I.; Moinard, C.; Osowska, S.; Zerrouk, N.; Bénazeth, S.; Cynober, L. Almost All about Citrulline in Mammals. Amino Acids 2005, 29, 177-205.

(47) Allerton, T. D.; Proctor, D. N.; Stephens, J. M.; Dugas, T. R.; Spielmann, G.; Irving, B. A. LCitrulline Supplementation: Impact on Cardiometabolic Health. Nutrients 2018, 10, 1-24.

(48) Gostner, J. M.; Becker, K.; Croft, K. D.; Woodman, R. J.; Puddey, I. B.; Fuchs, D.; Hodgson, J. M. Regular Consumption of Black Tea Increases Circulating Kynurenine Concentrations: A Randomized Controlled Trial. BBA Clin. 2015, 3, 31-35. 
599 (49) Keszthelyi, D.; Troost, F. J.; Masclee, A. A. M. Understanding the Role of Tryptophan and 600 Serotonin Metabolism in Gastrointestinal Function. Neurogastroenterol. Motil. 2009, 21, $601 \quad 1239-1249$.

602 (50) Zapata, R. C.; Singh, A.; Ajdari, N. M.; Chelikani, P. K. Dietary Tryptophan Restriction 603 Dose-Dependently Modulates Energy Balance, Gut Hormones, and Microbiota in Obesity$604 \quad$ Prone Rats. Obesity 2018, 26, 730-739. 
606

607

608

\section{Figure captions}

Figure 1. Experimental design. The nutritional intervention lasted for 7 or 18 days. After these periods, rats were deprived of food for $18 \mathrm{~h}$ and were then euthanized.

Figure 2. Mean motor activity from the three groups of study (white bars represent reference group (RF), grey bars represent cocoa group (CC), and striped bars represent theobromine group (TB)); during days 5 to $7(\mathrm{a}-\mathrm{b})$ and days 9 to $14(\mathrm{c}-\mathrm{d})$. Values are expressed as mean \pm standard error $(\mathrm{n}=$ 6-7). Statistical difference: ${ }^{*} \mathrm{p} \leq 0.05$ vs RF group by one-way ANOVA.

Figure 3. Fecal fat content and $\mathrm{pH}$ from the three groups of study (white bars represent reference group (RF), grey bars represent cocoa group (CC), and striped bars represent theobromine group (TB)). Values are expressed as mean \pm standard error $(n=6-7)$. Statistical difference: $* p \leq 0.05$ vs RF group, and $\delta \mathrm{p} \leq 0.05$ vs CC group by one-way ANOVA.

Figure 4. Liver gene expression. Liver gene expression from the three groups of study (white bars represent reference group (RF), grey bars represent cocoa group (CC); and striped bars represent theobromine group (TB)) in the 7 days-lasting experimental design. Values are expressed as mean \pm standard error ( $n=6-7)$. Statistical difference: * $p \leq 0.05$ vs RF group, and $\delta p \leq 0.05$ vs CC group by Mann-Whitney U test.

Figure 5. Adipose tissue gene expression. Liver gene expression from the three groups of study (white bars represent reference group (RF); grey bars represent cocoa group (CC); and striped bars represent theobromine group (TB) in the 7-day experimental design. Values are expressed as mean \pm standard error $(n=6-7)$. Statistical difference: ${ }^{*} p \leq 0.05$ vs RF group by Mann-Whitney U test.

Figure 6. Metabolic variables. Ghrelin, glucagon and insulin concentration from the three groups of study (white bars represent reference group (RF), grey bars represent cocoa group (CC), and striped bars represent theobromine group (TB)) in the 19-day experimental design. Values are expressed as mean \pm standard error $(n=6-7)$. Statistical difference: $* \mathrm{p} \leq 0.05$ vs RF group by one-way ANOVA. 
630 Figure 7. OSC-PLS-DA scores plots (PC1 versus PC2) of study samples. Plasma samples from 631 reference group are indicated in blue, cocoa group in yellow and theobromine group in red.

632 Figure 8. Tentatively identified metabolites. Plasma concentrations of exogenous (theobromine) 633 and endogenous (pantothenic acid, glycocholic acid, citrulline, CEDHP and HIAA) metabolites in 634 the three groups of study (white bars represent reference group (RF), grey bars represent cocoa 635 group (CC); and striped bars represent theobromine group (TB)) in the 7-day experimental design. 636 Values are expressed as mean \pm standard error $(n=6-7)$. Statistical difference: ${ }^{*} \mathrm{p} \leq 0.05$ vs $\mathrm{RF}$ 637 group by one-way ANOVA.

Figure 9. Heatmap representation of the association between metabolites (in rows) and some metabolic and biochemical variables (in columns). Blue color indicates a positive correlation and red color displays a negative correlation. Color intensity and circle size are proportional to the correlation coefficients. All displayed correlations were statistically significant (FDR $<0.05)$. 
Table 1. Morphometric measurements. Initial body weight, body weight gain, body length (with and without tail), body mass index, Lee's index, mean food and water intake, food efficiency, rectal temperature and the relative weight of inguinal and retroperitoneal fat, heart, liver, kidney, small intestine and colon and caecum in the two study time points and from the three groups of study (reference group (RF); , cocoa group (CC), and theobromine group (TB)). Values are expressed as mean \pm standard error $(\mathrm{n}=6-7)$. Statistical difference: ${ }^{*} \mathrm{p} \leq 0.05$ vs $\mathrm{RF}$ group, and $\delta \mathrm{p} \leq 0.05 \mathrm{vs} \mathrm{CC}$ group by one-way ANOVA.

\begin{tabular}{|c|c|c|c|c|c|c|}
\hline \multirow[b]{2}{*}{ Variable } & \multicolumn{3}{|c|}{ 7-days diet group } & \multicolumn{3}{|c|}{ 18-days diet group } \\
\hline & RF & $\mathbf{C C}$ & TB & RF & $\mathbf{C C}$ & TB \\
\hline Initial body weight (g) & $80.08 \pm 2.25$ & $79.98 \pm 1.86$ & $80.62 \pm 2.17$ & $80.23 \pm 2.81$ & $79.82 \pm 1.99$ & $79.22 \pm 1.14$ \\
\hline Body weight gain (g) & $35.30 \pm 1.61$ & $23.43 \pm 0.60^{*}$ & $23.03 \pm 0.88^{*}$ & $80.90 \pm 2.98$ & $51.53 \pm 0.65^{*}$ & $52.11 \pm 5.31 *$ \\
\hline Body length (cm) & $28.22 \pm 0.32$ & $27.90 \pm 0.29$ & $27.70 \pm 0.12$ & $29.51 \pm 0.28$ & $26.91 \pm 0.23^{*}$ & $27.10 \pm 0.52 *$ \\
\hline Body length without tail $(\mathrm{cm})$ & $15.03 \pm 0.16$ & $15.23 \pm 0.16$ & $14.98 \pm 0.14$ & $16.50 \pm 0.14$ & $14.96 \pm 0.17 *$ & $14.97 \pm 0.23^{*}$ \\
\hline Body mass index & $0.13 \pm 0.00$ & $0.11 \pm 0.00^{*}$ & $0.12 \pm 0.00^{*}$ & $0.11 \pm 0.01$ & $0.10 \pm 0.00^{*}$ & $0.10 \pm 0.00 *$ \\
\hline Lee's index & $3.11 \pm 0.00$ & $2.93 \pm 0.00^{*}$ & $2.99 \pm 0.00^{*}$ & $2.81 \pm 0.00$ & $2.78 \pm 0.00$ & $2.79 \pm 0.00$ \\
\hline Mean food intake (g/100 g rat/day) & $15.34 \pm 0.24$ & $16.27 \pm 0.24^{*}$ & $15.11 \pm 0.14^{\delta}$ & $13.75 \pm 0.15$ & $13.77 \pm 0.22$ & $13.46 \pm 0.20$ \\
\hline Mean water intake ( $\mathrm{g} / 100 \mathrm{~g}$ rat/day) & $12.20 \pm 0.70$ & $30.10 \pm 1.88^{*}$ & $27.93 \pm 2.78^{*}$ & $11.28 \pm 0.18$ & $20.54 \pm 0.41 *$ & $17.67 \pm 1.17 * \delta$ \\
\hline Food efficiency & $0.34 \pm 0.01$ & $0.30 \pm 0.01 *$ & $0.31 \pm 0.01 *$ & $0.35 \pm 0.01$ & $0.30 \pm 0.01 *$ & $0.30 \pm 0.00 *$ \\
\hline Rectal temperature $\left({ }^{\circ} \mathrm{C}\right)$ & $35.85 \pm 0.13$ & $36.12 \pm 0.11$ & $36.03 \pm 0.17$ & $36.91 \pm 0.34$ & $37.3 \pm 0.26$ & $36.49 \pm 0.30$ \\
\hline
\end{tabular}

Relative weight (\%) 


\begin{tabular}{|c|c|c|c|c|c|c|}
\hline Inguinal fat & $0.15 \pm 0.02$ & $0.13 \pm 0.01$ & $0.11 \pm 0.01$ & $0.29 \pm 0.03$ & $0.29 \pm 0.03$ & $0.19 \pm 0.02 * \delta$ \\
\hline Retroperitoneal fat & $0.13 \pm 0.02$ & $0.05 \pm 0.02 *$ & $0.07 \pm 0.02 *$ & $0.11 \pm 0.01$ & $0.04 \pm 0.01 *$ & $0.03 \pm 0.00^{*}$ \\
\hline Heart & $0.55 \pm 0.02$ & $0.57 \pm 0.02$ & $0.54 \pm 0.02$ & $0.58 \pm 0.01$ & $0.65 \pm 0.02 *$ & $0.59 \pm 0.02$ \\
\hline Liver & $3.82 \pm 0.05$ & $3.66 \pm 0.09$ & $3.66 \pm 0.05$ & $4.12 \pm 0.06$ & $3.80 \pm 0.05^{*}$ & $3.46 \pm 0.17 * \delta$ \\
\hline Kidney (one) & $0.48 \pm 0.02$ & $0.57 \pm 0.03$ & $0.52 \pm 0.01$ & $0.49 \pm 0.01$ & $0.51 \pm 0.01$ & $0.52 \pm 0.03$ \\
\hline Small intestine & $3.31 \pm 0.03$ & $3.67 \pm 0.07 *$ & $3.62 \pm 0.05^{*}$ & $4.19 \pm 0.12$ & $4.80 \pm 0.20^{*}$ & $4.83 \pm 0.14^{*}$ \\
\hline Colon and caecum & $1.49 \pm 0.05$ & $2.42 \pm 0.13^{*}$ & $2.66 \pm 0.13^{*}$ & $0.98 \pm 0.03$ & $1.36 \pm 0.03^{*}$ & $1.61 \pm 0.06^{* \delta}$ \\
\hline
\end{tabular}

Table 2. Biochemical variables measurements. Fold increase of total cholesterol, HDL-c, LDL-c, serum and hepatic TG, and glucose from the three groups of study (reference group (RF), cocoa group (CC), theobromine group (TB)) at day 8 and 19. Values are expressed as mean \pm standard error (n =6-7). Statistical difference: * $\mathrm{p} \leq 0.05$ vs RF group by one-way ANOVA.

\begin{tabular}{|c|c|c|c|c|c|c|}
\hline \multirow[b]{2}{*}{ Variable } & \multicolumn{3}{|l|}{ 7-days effect } & \multicolumn{2}{|c|}{ 18-days effect } & \multirow[b]{2}{*}{ TB } \\
\hline & RF & $\mathbf{C C}$ & TB & RF & $\mathrm{CC}$ & \\
\hline Total cholesterol & $1.00 \pm 0.06$ & $0.85 \pm 0.03 *$ & $0.86 \pm 0.02 *$ & $1.00 \pm 0.01$ & $1.04 \pm 0.03$ & $0.98 \pm 0.02$ \\
\hline HDL-c & $1.00 \pm 0.08$ & $1.41 \pm 0.07^{*}$ & $1.39 \pm 0.08^{*}$ & $1.00 \pm 0.05$ & $1.34 \pm 0.05^{*}$ & $1.24 \pm 0.09 *$ \\
\hline LDL-c & $1.00 \pm 0.07$ & $0.76 \pm 0.04 *$ & $0.78 \pm 0.03^{*}$ & $1.00 \pm 0.01$ & $1.00 \pm 0.03$ & $0.95 \pm 0.03$ \\
\hline Plasma TG & $1.00 \pm 0.08$ & $1.17 \pm 0.09$ & $1.13 \pm 0.12$ & $1.00 \pm 0.11$ & $0.83 \pm 0.07$ & $0.54 \pm 0.05^{*}$ \\
\hline Hepatic TG & $1.00 \pm 0.02$ & $0.35 \pm 0.10^{*}$ & $0.12 \pm 0.06^{*}$ & $1.00 \pm 0.10$ & $0.35 \pm 0.02 *$ & $0.34 \pm 0.03 *$ \\
\hline Glucose & $1.00 \pm 0.06$ & $1.10 \pm 0.07$ & $1.02 \pm 0.06$ & $1.00 \pm 0.23$ & $2.09 \pm 0.40^{*}$ & $2.45 \pm 0.43^{*}$ \\
\hline
\end{tabular}


Standard, $10 \%$ cocoa or $0.25 \%$ teobromine

Standard, $10 \%$ cocoa or $0.25 \%$ teobromine

\begin{tabular}{|l|l|l|l|l|l|l|l|l|l|l|l|l|l|l|l|l|l|l|l|}
\hline 0 & 1 & 2 & 3 & 4 & 5 & 6 & 7 & 8 & 9 & 10 & 11 & 12 & 13 & 14 & 15 & 16 & 17 & 18 & 19 \\
\hline
\end{tabular}

MOTOR ACTIVITY

7-day diet group

$\checkmark$ Morphometric assessment

$\checkmark$ Biochemical measurements

$\checkmark$ Fecal fat and $\mathrm{pH}$

$\checkmark$ Gene expression

$\checkmark$ Metabolomic analysis

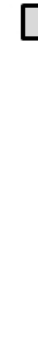

MOTOR ACTIVITY

\begin{tabular}{|l|}
\hline \multicolumn{2}{|c|}{ 18-day diet group } \\
\hline$\checkmark$ Morphometric assessment \\
$\checkmark$ Biochemical measurements \\
$\checkmark$ Fecal pH \\
\\
\hline
\end{tabular}

Figure 1

$321 \times 143 \mathrm{~mm}(72 \times 72 \mathrm{DPI})$ 

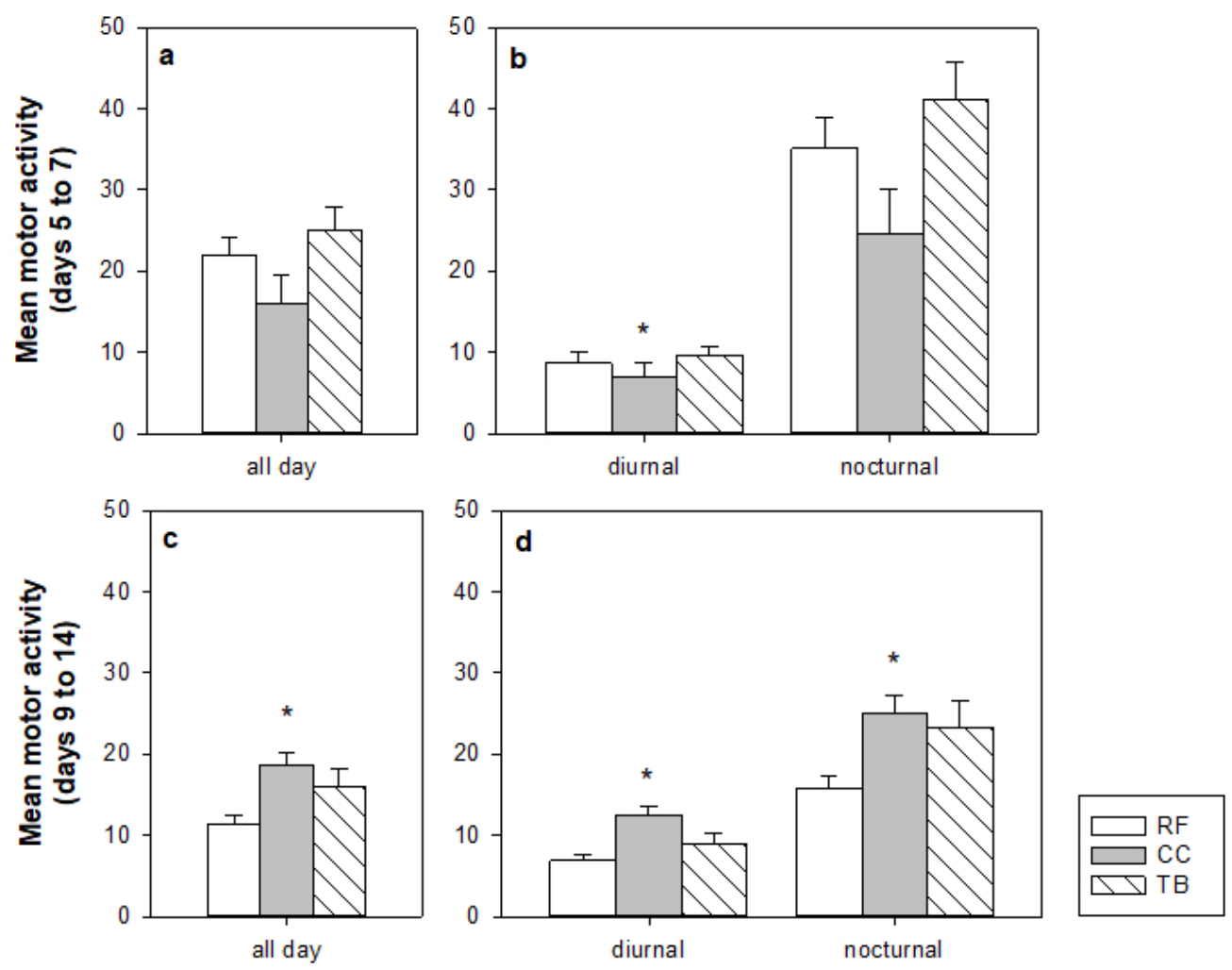

Figure 2

$242 \times 200 \mathrm{~mm}(72 \times 72$ DPI $)$ 

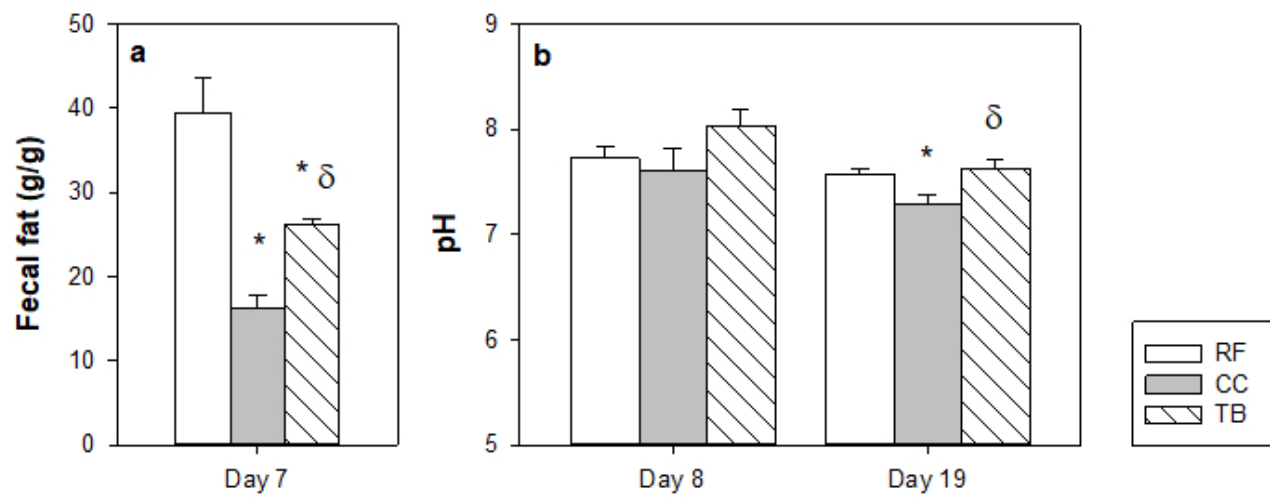

Figure 3

$247 \times 104 \mathrm{~mm}$ (72 x 72 DPI) 

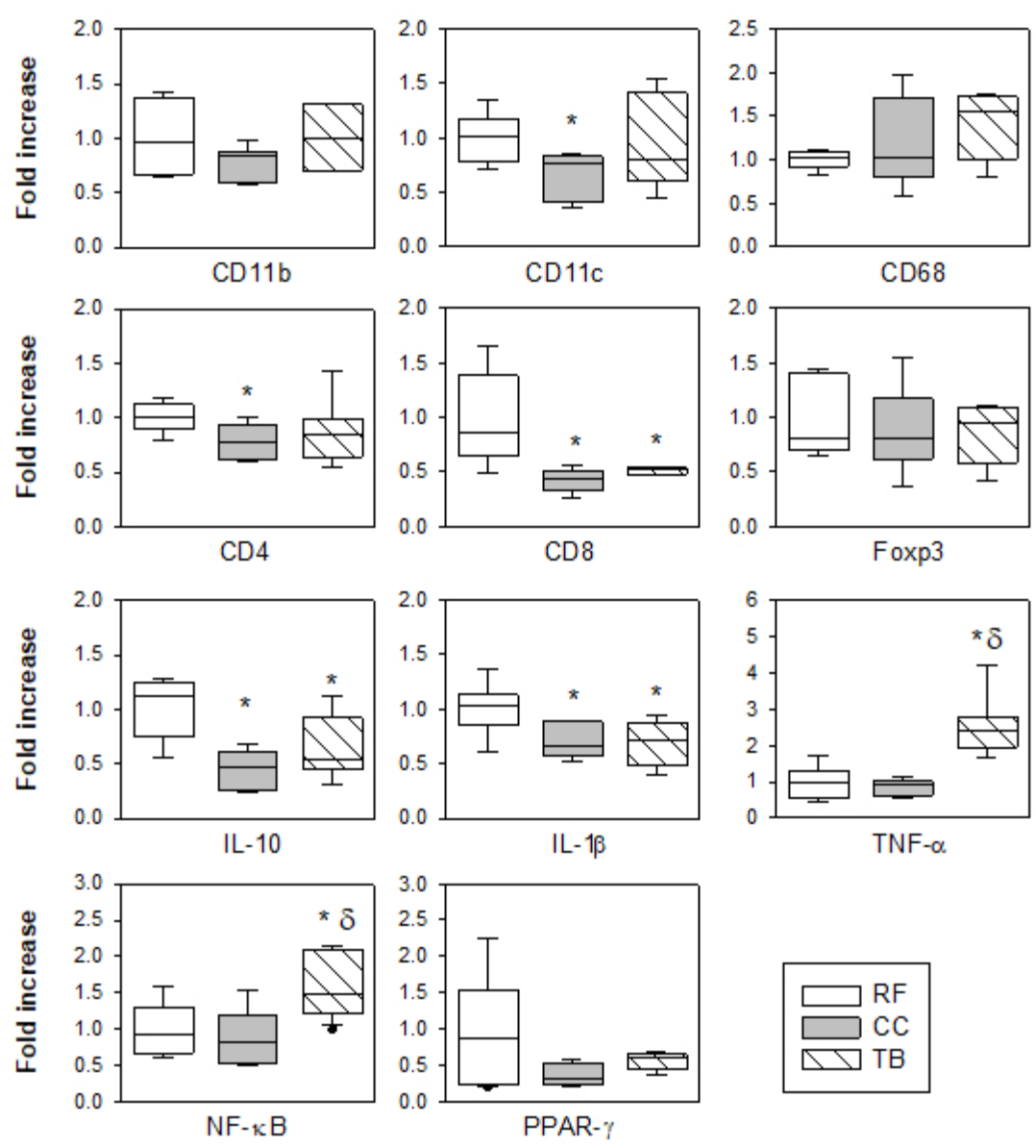

Figure 4

$230 \times 222 \mathrm{~mm}(72 \times 72 \mathrm{DPI})$ 

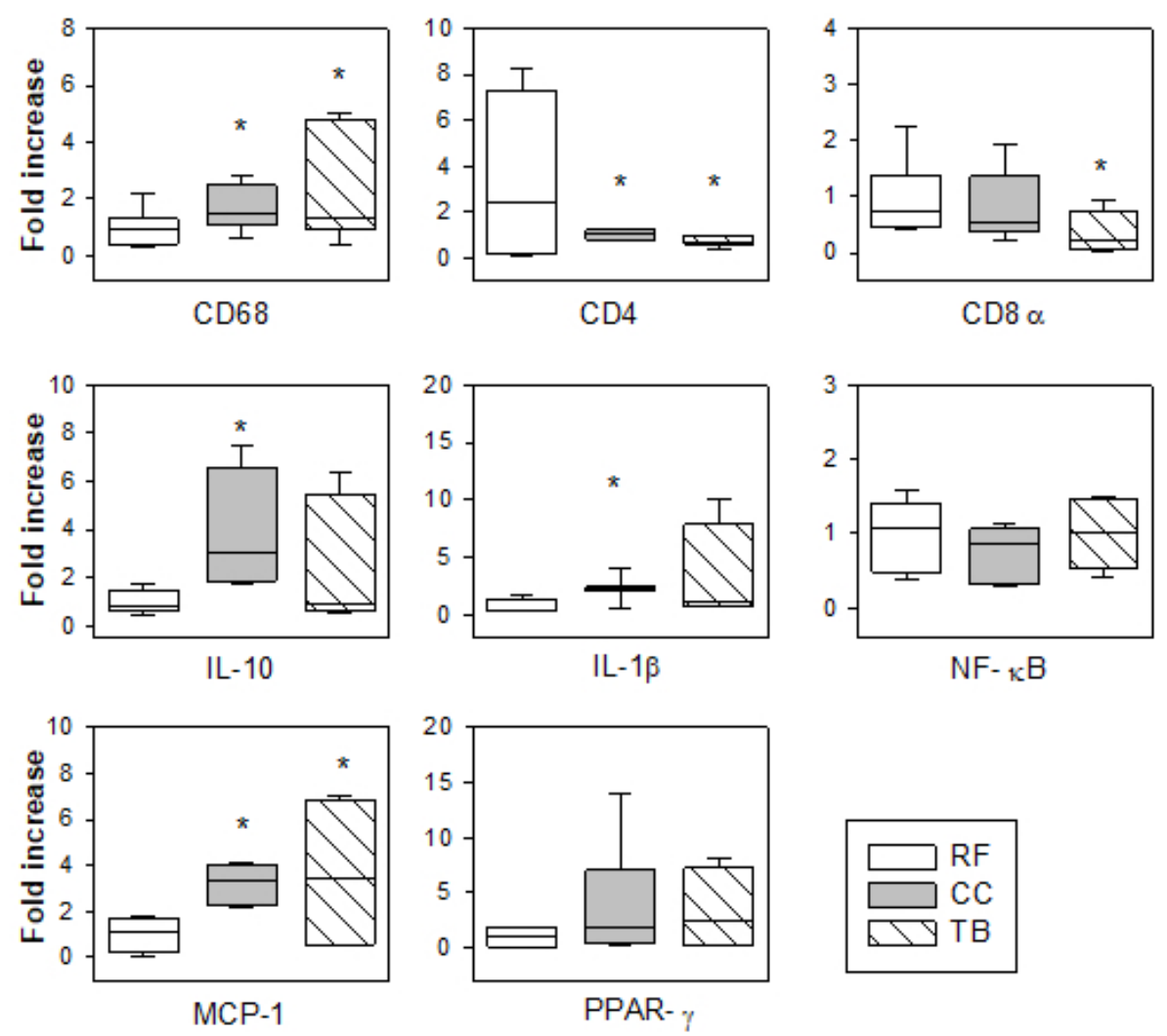

Figure 5

$201 \times 164 \mathrm{~mm}(72 \times 72 \mathrm{DPI})$ 

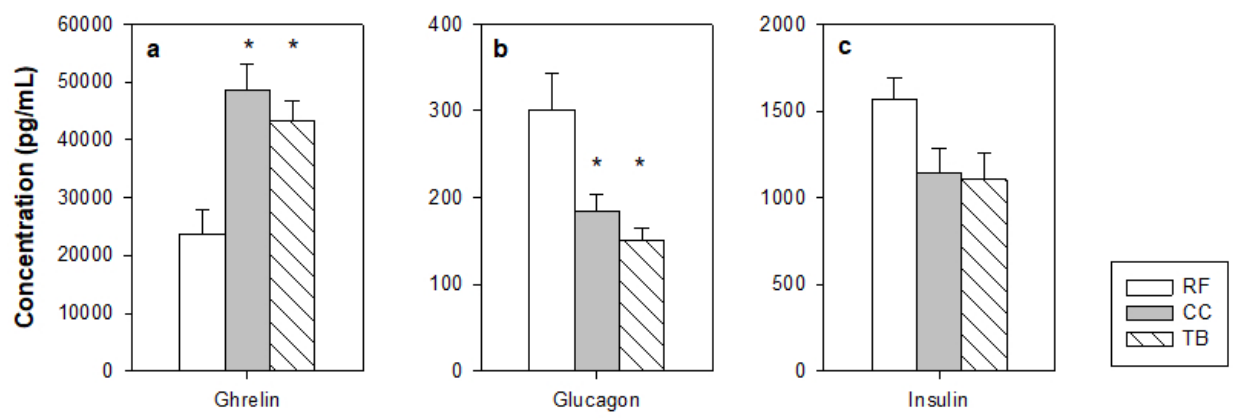

Figure 6

$299 \times 128 \mathrm{~mm}(72 \times 72 \mathrm{DPI})$ 


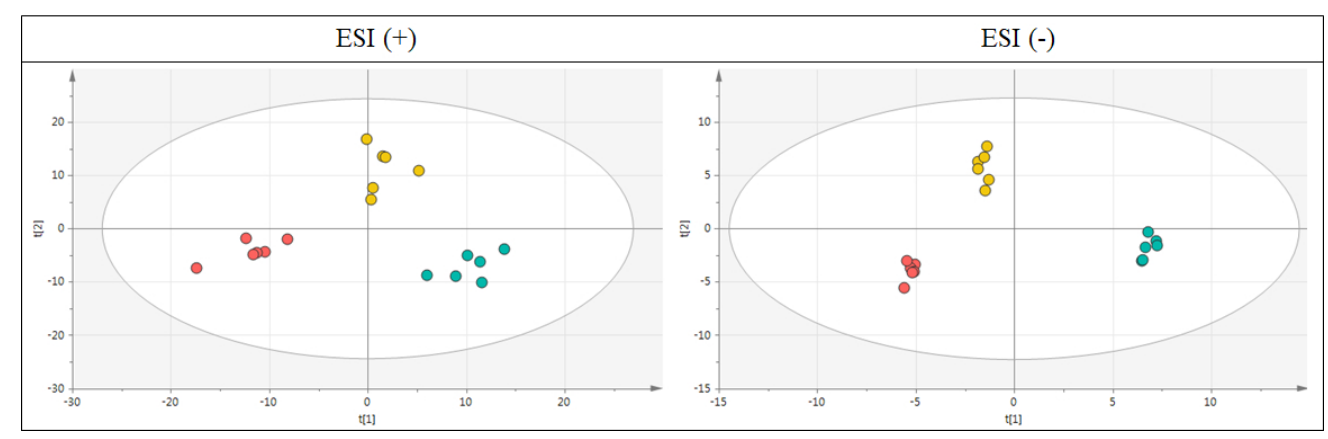

Figure 7

$408 \times 130 \mathrm{~mm}(72 \times 72$ DPI $)$ 

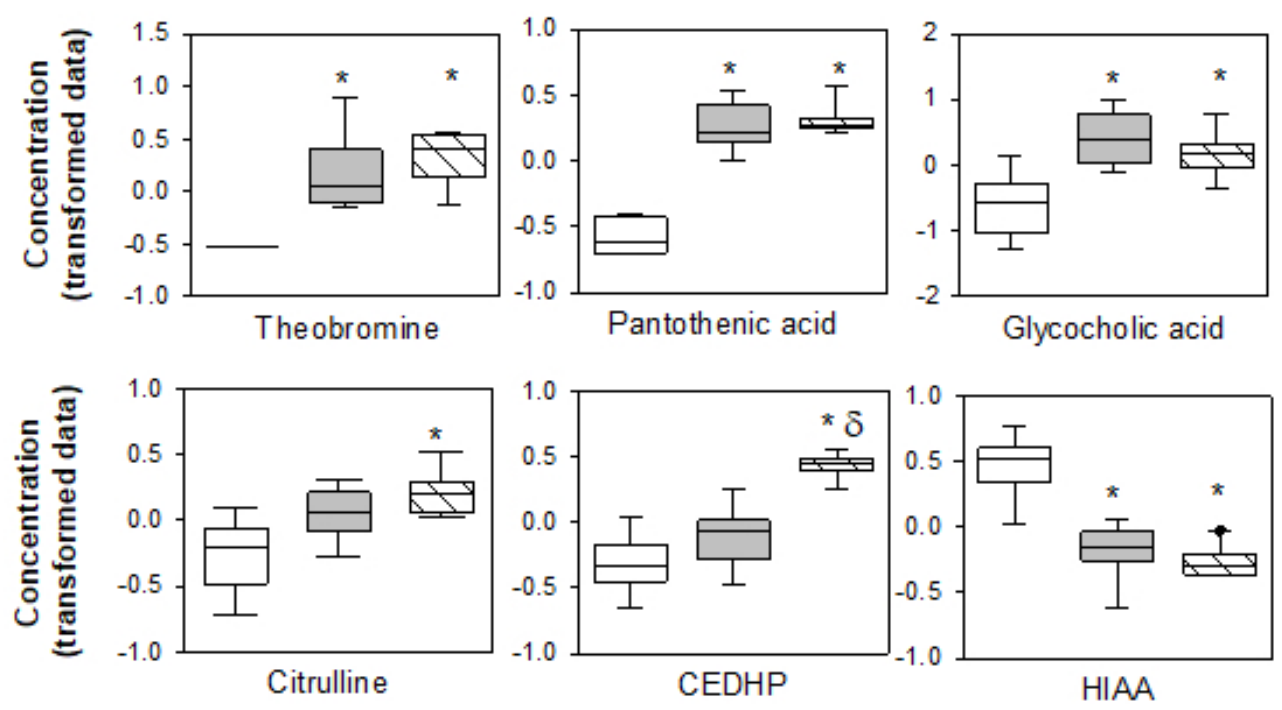

Figure 8

$196 \times 109 \mathrm{~mm}(72 \times 72 \mathrm{DPI})$ 


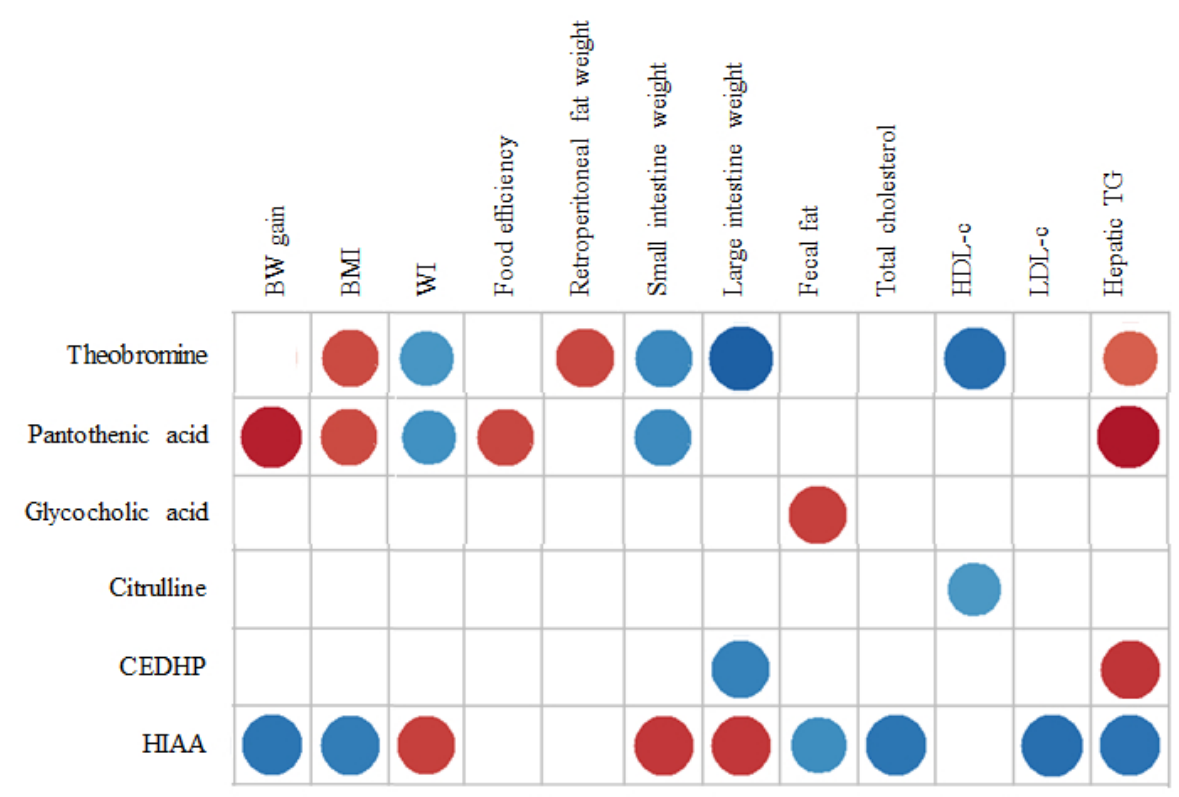

Figure 9

$273 \times 178 \mathrm{~mm}(72 \times 72 \mathrm{DPI})$ 